\title{
A REMARK ON THE CRITICAL EXPLOSION PARAMETER FOR A SEMILINEAR ELLIPTIC EQUATION IN A GENERIC DOMAIN USING AN EXPLOSION TIME OF AN ORDINARY DIFFERENTIAL EQUATION
}

\author{
J. Dolbeault $(*)$ \\ CEREMADE (U.R.A. 749) \\ Université Paris IX - Dauphine \\ Place du Maréchal de Lattre de Tassigny \\ 75775 Paris Cédex 16, France \\ e-mail: jeandol@siberia.ups-tlse.fr \\ et \\ F. Poupaud \\ Laboratoire J.A. Dieudonné (U.R.A. 168) \\ Université de Nice Sophia -Antipolis \\ Parc Valrose, B.P. 71 \\ 06108 Nice Cédex 02, France \\ e-mail: poupaud@math.unice.fr
}

\section{Key-words}

Nonlinear eigenvalue problems - Semilinear elliptic problems - Surlinear nonlinearities - Critical explosion parameter - Existence results - Nonexistence results - Poisson problem - Ordinary differential equations - Explosion time - Nonlinear Dirichlet problem - Isoperimetric inequalities - Rearrangements - Schwarz' symmetrisation - Shooting method - Radially symmetric solutions - Maximum principle - Weight functions - Non autonomous nonlinear scalar field equations

Mathematics Subject Classification : Primary : 35P30; Secondary : 34A34, 35J15, 35J60

\begin{abstract}
This paper gives a new criterion for estimating the critical explosion parameter of a semilinear elliptic equation with Dirichlet conditions on a bounded or unbounded domain, using simple evaluations of the geometric part of the problem on one hand, and of an explosion time for a first order ordinary differential equation on the other hand. This criterion is evaluated in the special case of a ball, and compared with other usual criteria deduced from the properties of the first eigenfunction of the laplacien, and from Pohozaev's method.
\end{abstract}

\section{Contents}

1. Introduction

2. An upper bound for the critical explosion parameter using ordinary differential equations

3. The radial case

4. Comparison with other usual criteria

4.1 A bound deduced from the comparison with a principal eigenvalue

4.2 A bound deduced from Pohozaev's method

Conclusion

References

(*) and Groupe de Physique Théorique, Laboratoire de Physique Quantique (U.R.A. 505), IRSAMC, Université Paul Sabatier, 118, route de Narbonne, 31062 Toulouse Cédex, France 


\section{Introduction}

Let $\Omega$ be a $C^{1}$ domain in $\mathbb{R}^{N}(N \geq 1), \rho$ be a strictly positive $L_{\text {loc }}^{2}$ function, and consider in $H_{0}^{1}(\Omega)$ the following nonlinear eigenvalue problem

$$
-\Delta U=\lambda \cdot \rho(x) f(U)
$$

If we assume that

$$
f \text { is a Lipshitz non decreasing function on } \mathbb{R}^{+} \text {such that } f(0)>0
$$

then it is a well known result that, if $\Omega$ is bounded and $\rho$ belongs to $L^{\infty}(\Omega)$, there exists a $\lambda_{c r}>0$ such that 1) for each $\lambda \in\left(0, \lambda_{c r}\right)$, equation (1) has at least one solution in $H_{0}^{1}(\Omega)$; this solution belongs to $L^{\infty}(\Omega)$ (for regularity results, see [ADN], [Mor])

2) equation (1) has no solution for $\lambda>\lambda_{c r}$

(see for example [A1], [A2], [B1], [CR], [FLN1,2], [GS], [J], [L], [JL], [KC], [Lio].) These results can be extended to many (more technical) cases where $\Omega$ is unbounded, $\rho$ does not belong to $L^{\infty}(\Omega)$ and the laplacian is replaced by a more general elliptic operator. $\lambda_{c r}$ is called the critical explosion parameter.

In this paper, we will give estimates on $\lambda_{c r}$ using a new point of view which separates clearly what arises from the nonlinearity of $f$, and is caracterised by the explosion time of a first order ordinary differential equation, and what arises from the geometry of the domain and from the measure $\rho(x) d x$. In Section 2, we shall state our main result, giving an upper bound for the critical explosion parameter $\lambda_{c r}$. The method also provides a necessary condition for the existence of a solution of equation (1). It is based on an estimate (from below) of the solution, which in a sense gives a refinement of the Maximum Principle in the case of semilinear elliptic equations with a nonlinear (and eventually non autonomous) term. The proof relies on a "first order approximation" of the solution along the level surfaces of a linear problem. It is a kind of rearrangement method (for general rearrangement techniques, see [B1], [HLP], [Mos] for instance).

In Section 3, we will focus on the radial case and explain more in details the strategy developped in Section 2. The comparison with the "first order approximation", explicitly written using a change of variables (the rearrangement here is obvious, since the functions are monotones), will explain why the criterion is not optimal. Section 4 is devoted to the comparison with two other common criteria for getting upper bounds for the critical explosion parameter. Finally, let us just mention that the interest of our method lies in the fact that it is easier to use than criteria deduced from Pohozaev's method, and also takes the full nonlinearity, and not only the value of an infimum (as it is the case for the method deduced from the comparison with a principal eigenvalue), into account. The method works for a bounded domain as well as for an unbounded one, and could be extended to more general Dirichlet boundary conditions, elliptic operators, etc (see the conclusion).

\section{An upper bound for the critical explosion parameter using ordinary differential equations}

Let us consider two different problems. Let $\psi$ be the solution in $H_{0}^{1}(\Omega)$ of the homogeneous Dirichlet problem for the Poisson equation

$$
-\Delta \psi=\rho
$$

and $t \mapsto y(t)$ the solution of

$$
\left\{\begin{array}{l}
\frac{d y}{d t}(t)=f(y(t)) \\
y(0)=0
\end{array}\right.
$$

We shall assume that $\Omega$ and $\rho$ are choosen in a way such that

$$
\psi \in H_{0}^{1}(\Omega) \cap C_{0}^{1}(\Omega)
$$


Here $\psi \in C_{0}^{1}(\Omega)$ means : $\psi \in C^{1}(\Omega)$ and $\lim _{|x| \rightarrow+\infty, x \in \Omega} \psi(x)=0$ (if $\Omega$ is unbounded). This regularity condition is choosen in order to allow us to apply the usual Maximum Principle (to the domain $\Omega_{\tau}$ : see below), but could probably be weakned a little bit (see [BNV] for Maximum Principles in general domains). Condition ( $H 2$ ) is automatically satisfied if, for instance, $\Omega$ is a bounded regular domain in $\mathbb{R}^{2}$. Now, let us state our main result.

Theorem 1 : Let $\Omega$ be a $C^{1}$ domain of $\mathbb{R}^{N}(N \geq 1), \rho$ be a strictly positive $L_{\text {loc }}^{2}$ function on $\Omega$, and let us assume that $f, \rho$ and $\Omega$ satisfy assumptions (H1) and (H2). Then we have the following estimate

$$
\lambda_{c r} \leq \frac{T(f)}{M(\Omega, \rho)}
$$

where

$$
M(\Omega, \rho)=\sup _{x \in \Omega} \psi(x)
$$

( $\psi$ is the solution of equation $(2)$ ) and $T(f) \in] 0,+\infty]$ is the maximal existence time of the solution of equation (3).

The proof is an immediate consequence of the

Lemma 2 : Under the same assumptions as in Theorem 1, for any $\lambda>0$, if equation (1) has a solution, then

$$
M(\Omega, \rho)<\frac{T(f)}{\lambda}
$$

and

$$
y(\tau) \leq \inf _{x \in \Omega, \psi(x)=\tau} U(x) \quad \forall \tau \in[0, \lambda M(\Omega, \rho)]
$$

so that

$$
y(\lambda M(\Omega, \rho)) \leq\|u\|_{L^{\infty}(\Omega)}
$$

Proof of Lemma 2 : For any $\tau \in] 0, m[$ with $m=\min (T(f) / \lambda, M(\Omega, \rho))$, let us define

$$
\begin{gathered}
\Omega_{\tau}=\{x \in \Omega \mid \psi(x)>\tau\} \\
z(\tau)=\inf _{x \in \Omega_{\tau}} U(x)
\end{gathered}
$$

where $U$ is the solution of equation (1). Let $v_{\tau}$ be the solution in $H^{1}\left(\Omega_{\tau}\right)$ of

$$
\left\{\begin{array}{l}
-\Delta v_{\tau}(x)=\lambda f(z(\tau)) \cdot \rho(x) \quad \forall x \in \Omega_{\tau} \\
v_{\tau \mid \partial \Omega_{\tau}}(x)=z(\tau) \quad \forall x \in \partial \Omega_{\tau}
\end{array}\right.
$$

$\Omega_{\tau}$ is a $C^{1}$ bounded domain because of $(H 2)$, and we can notice that

$$
v_{\tau}(x)=z(\tau)+\lambda f(z(\tau)) \cdot(\psi(x)-\tau) \quad \forall x \in \Omega_{\tau}
$$

Because of the Maximum Principle, the minimum of $U$ in $\overline{\Omega_{\tau}}$ belongs to $\partial \Omega_{\tau}$ :

$$
U(x) \geq \inf _{x \in \Omega_{\tau}} U(x)=\inf _{x \in \partial \Omega_{\tau}} U(x)=z(\tau)=v_{\tau \mid \partial \Omega_{\tau}}(x) \quad \forall x \in \partial \Omega_{\tau}
$$

and

$$
-\Delta U(x)=\lambda \cdot \rho(x) f(U(x)) \geq \lambda f(z(\tau)) \cdot \rho(x)=-\Delta v_{\tau}(x) \quad \forall x \in \Omega_{\tau}
$$


because according to $(H 1), t \mapsto f(t)$ is nondecreasing. According to the Maximum Principle,

$$
U(x) \geq v_{\tau}(x) \quad \forall x \in \Omega_{\tau}
$$

Let us choose $\delta \tau>0$. Using the definition of $\Omega_{\tau}$, we get

$$
\begin{gathered}
z(\tau+\delta \tau)=\inf _{x \in \partial \Omega_{(\tau+\delta \tau)}} U(x)=\inf _{x \in \Omega_{(\tau+\delta \tau)}} U(x) \geq \inf _{x \in \Omega_{(\tau+\delta \tau)}} v_{\tau}(x)=\inf _{x \in \partial \Omega_{(\tau+\delta \tau)}} v_{\tau}(x)=\lambda f(z(\tau)) \cdot \delta \tau+z(\tau) \\
\frac{1}{\delta \tau}(z(\tau+\delta \tau)-z(\tau)) \geq \lambda f(z(\tau))
\end{gathered}
$$

Of course $z(0)=0$, and therefore, if $y$ is the solution of equation (3), then

for all $\tau \in[0, m=\min (T(f) / \lambda, M(\Omega, \rho))[$

$$
z(\tau) \geq y(\lambda \tau)
$$

which proves that

$$
y(\lambda \tau) \leq \inf _{x \in \Omega_{\tau}} U(x) \leq\|U\|_{L^{\infty}(\Omega)} \quad \forall \tau \in[0, m[
$$

$T(f) \leq \lambda M(\Omega, \rho)$ is impossible, because in this case, we would have

$$
+\infty=\lim _{\tau \rightarrow(T(f) / \lambda)_{-}} y(\lambda \tau) \leq\|U\|_{L^{\infty}(\Omega)}
$$

Therefore $M(\Omega, \rho)<T(f) / \lambda$ and we have

$$
y(\lambda M(\Omega, \rho)) \leq \lim _{\tau \rightarrow M(\Omega, \rho)_{-}} \inf _{x \in \Omega_{\tau}} U(x) \leq\|U\|_{L^{\infty}(\Omega)}
$$

\section{The radial case}

In this section, we will explain more in details what happens in the simpler case of a ball. The simplification here is due to the fact that the functions are all radial : one has to deal with their value (on a sphere), and not the values of infima taken on the boundary of a domain $\left(\partial \Omega_{\tau}\right.$ with the notations of the previous section). This explain why we can write for these functions explicit equations after appropriate changes of variables.

Let us assume that $\Omega=B(R)$ is a ball of radius $R$ and center 0 , that $\rho$ is a radial, bounded, strictly positive decreasing function and that $f$ is of class $C^{1}$. (We shall use the non rigourous notation $\rho(x)=\rho(|x|)$ ). According to the results contained in the Gidas, Ni and Nirenberg famous paper (see [GNN1]), any solution $U$ of equation (1) is radial and therefore $u(|x|)=U(x)$ satisfies

$$
\left\{\begin{array}{l}
-\frac{1}{r^{N-1}} \frac{d}{d r}\left(r^{N-1} \frac{d u}{d r}(r)\right)=\lambda \cdot \rho(r) f(u(r)) \\
\frac{d u}{d r}(0)=0 \\
u(R)=0
\end{array}\right.
$$

The solution $\psi$ of

$$
\left\{\begin{array}{l}
-\frac{1}{r^{N-1}} \frac{d}{d r}\left(r^{N-1} \frac{d \psi}{d r}(r)\right)=\rho(r) \\
\frac{d \psi}{d r}(0)=0 \\
\psi(R)=0
\end{array}\right.
$$

is stricly decreasing on $] 0, R]$ because

$$
\frac{d \psi}{d r}(r)=-\frac{1}{r^{N-1}} \int_{0}^{r} t^{N-1} \rho(t) d t
$$


For all $\tau \in[0, \psi(0)]$, let us define

$$
w(\tau)=u\left(\psi^{-1}(\tau)\right)
$$

$w$ satisfies

$$
\left\{\begin{array}{l}
\frac{d w}{d \tau}(\tau)-\lambda f(w(\tau))=\frac{\left(\frac{d w}{d r}\left(\psi^{-1}(\tau)\right)\right)^{2}}{\rho\left(\psi^{-1}(\tau)\right)} \cdot \frac{d^{2} w}{d \tau^{2}}(\tau) \\
w(0)=0 \\
\frac{d w}{d \tau}(0)=p
\end{array}\right.
$$

where $p$ is defined by

$$
p=\frac{\frac{d u}{d r}(R)}{\frac{d \psi}{d r}(R)}=\lambda \cdot \frac{\int_{0}^{R} r^{N-1} \rho(r) f(u(r)) d r}{\int_{0}^{R} r^{N-1} \rho(r) d r}
$$

or

$$
p=\lambda \cdot \frac{\int_{0}^{\psi(0)}\left(\psi^{-1}(\tau)\right)^{N-1} \rho\left(\psi^{-1}(\tau)\right) f(w(\tau)) \cdot\left(\frac{d \psi}{d r}\left(\psi^{-1}(\tau)\right)\right)^{-1} d \tau}{\int_{0}^{\psi(0)}\left(\psi^{-1}(\tau)\right)^{N-1} \rho\left(\psi^{-1}(\tau)\right) \cdot\left(\frac{d \psi}{d r}\left(\psi^{-1}(\tau)\right)\right)^{-1} d \tau}
$$

Conversely, if $w$ is a solution of equation (6) for some $p \in \mathbb{R}$ on the interval $[0, \psi(0)]$, then the condition

$$
\frac{d u}{d r}(0)=0
$$

is automatically satisfied because

$$
\frac{d u}{d r}(0)=\frac{d w}{d \tau}(\psi(0)) \cdot \frac{d \psi}{d r}(0) \text { and } \frac{d \psi}{d r}(0)=0
$$

We can now state the

Proposition 3 : Let us assume that $\rho$ is a radial bounded strictly positive nonincreasing function and such that the solution in $H_{0}^{1}(B(R))$ of equation (5) is bounded in $L^{\infty}\left(B(R)\right.$ ), that $f$ is of class $C^{1}$, nondecreasing on $\mathbb{R}^{+}$and such that $f(0)>0$. Then if equation (4) has a solution, there exists a solution $w$ of equation (6) on $[0, \psi(0)]$ satisfying condition (7), and we have the following properties

(i) $\frac{d w}{d \tau}(\psi(0))=\lambda f(w(\psi(0)))$

(ii) $\frac{d w}{d \tau}(\tau) \geq \lambda f(w(\tau)) \forall \tau \in[0, \psi(0)]$

(iii) $\frac{d w}{d \tau}(\tau)>\lambda f(w(\tau)) \forall \tau \in[0, \psi(0)$ [ if $f$ is strictly increasing

(iv) if $\frac{d w}{d \tau}(\tau)=\lambda f(w(\tau)) \forall \tau \in\left[0, \psi(0)\left[\right.\right.$, then $f$ is constant on $\left[0, \frac{\psi(0)}{\lambda f(0)}\right]$

\section{Proof of Proposition 3 :}

(i) is a straigtforward consequence of equations (5) and (6) : $\frac{d \psi}{d r}(0)=0$ implies

$$
\frac{\left(\frac{d \psi}{d r}\left(\psi^{-1}(\tau)\right)\right)^{2}}{\rho\left(\psi^{-1}(\tau)\right)} \cdot \frac{d^{2} w}{d \tau^{2}}(\tau)_{\mid \tau=\psi(0)}=0
$$

(ii) Let us assume that (ii) is false : there exists a $\tau_{0} \in[0, \psi(0)[$ such that

$$
\frac{d w}{d \tau}\left(\tau_{0}\right)<\lambda f\left(w\left(\tau_{0}\right)\right)
$$

$\frac{d^{2} w}{d \tau^{2}}(\tau)_{\mid \tau=\tau_{0}}<0$, and $w$ beeing of class $C^{2}$, there exists a neighbourhood $\left[\tau_{0}, \tau_{0}+\varepsilon\left[\right.\right.$ of $\tau_{0}+$ such that $\frac{d^{2} w}{d \tau^{2}}(\tau)<0$. Then it proves that $\tau \mapsto \frac{d w}{d \tau}(\tau)$ is strictly decreasing on $\left[\tau_{0}, \tau_{0}+\varepsilon\right.$. On the other hand, $\frac{d w}{d \tau}(\tau)>0$ for all $\left.\left.\tau \in\right] 0, \psi(0)\right]$ because

$$
\frac{d w}{d \tau}(\tau)=\frac{d}{d \tau}\left(\psi^{-1}(\tau)\right) \cdot\left(-\frac{\lambda}{r^{N-1}} \int_{0}^{r} t^{N-1} \rho(t) f(u(t)) d t\right)_{\mid r=\psi^{-1}(\tau)}>0
$$


This implies that $\tau \mapsto w(\tau)$ is increasing, and therefore $\tau \mapsto \frac{d w}{d \tau}(\tau)-\lambda f(w(\tau))$ is strictly decreasing on $\left[\tau_{0}, \tau_{0}+\varepsilon\left[\right.\right.$. Using the same arguments for $\tau=\tau_{0}+\varepsilon$, we prove that $\tau \mapsto \frac{d w}{d \tau}(\tau)-\lambda f(w(\tau))$ is strictly decreasing on $\left[\tau_{0}, \psi(0)\right]$ and therefore that $\frac{d w}{d \tau}(\psi(0))<\lambda f(w(\psi(0)))$, in contradiction with (i).

(iii) The proof of (iii) is similar to the proof of (ii). Let us assume that there exists a $\tau_{0} \in[0, \psi(0)$ [ such that

$$
\frac{d w}{d \tau}\left(\tau_{0}\right)=\lambda f\left(w\left(\tau_{0}\right)\right) \geq \lambda f(0)>0
$$

In a neighbourhood of $\tau_{0}+, \frac{d w}{d \tau}>0: \tau \mapsto \frac{d w}{d \tau}(\tau)-\lambda f(w(\tau))$ is strictly decreasing because $f$ is strictly increasing, and $\frac{d^{2} w}{d \tau^{2}}(\tau)$ is small because $w$ is of class $C^{2}$ and $\frac{d^{2} w}{d \tau^{2}}\left(\tau_{0}\right)=0$. Thus we get the same contradiction as before.

(iv) if $\frac{d w}{d \tau}(\tau)=\lambda f(w(\tau)) \forall \tau \in[0, \psi(0)]$, then $\frac{d^{2} w}{d \tau^{2}}(\tau)=0$ according to equation (6), and

$$
w(\tau)=p \tau \quad \forall \tau \in[0, \psi(0)]
$$

with the condition

$$
p-\lambda f(p \tau)=0 \quad \forall \tau \in[0, \psi(0)]
$$

so that

$$
\begin{gathered}
p=\lambda f(0), \quad \text { and } \quad f(0)-f(\lambda f(0) \tau)=0 \quad \forall \tau \in[0, \psi(0)] \\
f(u)=f(0) \quad \forall u \in\left[0, \frac{\psi(0)}{\lambda f(0)}\right]
\end{gathered}
$$

Proposition 3 allows us to give a new interpretation of Theorem 1. Let us summerize our results in the

\section{Remark 4 :}

(i) Proposition 3 provides an other proof of Theorem 1 (in the radial case) :

$$
\left.\|U\|_{L^{\infty}(B(R))}=u(0)=w(\psi(0)) \geq y(\lambda \psi(0))=y(\lambda M(B(R), \rho))\right)
$$

Moreover the inequality is strict provided that $f$ is not constant on a neighbourhood of $0+$. In this last case, we have

$$
\left.\left.y(\tau)<\inf _{x \in \Omega, \psi(x)=\tau} U(x) \quad \forall \tau \in\right] 0, \lambda M(\Omega, \rho)\right]
$$

and

$$
y(\lambda M(\Omega, \rho))<\|u\|_{L^{\infty}(\Omega)}
$$

(ii) Proposition 3 gives a necessary condition for the existence of a solution of equation (4) : with the same conditions as in Proposition 3, if equation (4) has a solution, then

$$
T_{f}(\lambda)>M(B(R), \rho)
$$

where $T_{f}(\lambda)=\sup _{q>0} T_{f}(\lambda, q, R, \rho)$, and $T_{f}(\lambda, q, R, \rho)$ is the maximal existence time of the solution of

$$
\left\{\begin{array}{l}
\frac{d w}{d \tau}(\tau)-\lambda f(w(\tau))=\frac{\left(\frac{d \psi}{d r}\left(\psi^{-1}(\tau)\right)\right)^{2}}{\rho\left(\psi^{-1}(\tau)\right)} \frac{d^{2} w}{d \tau^{2}} \\
\frac{d w}{d \tau}(0)=q \\
w(0)=0
\end{array}\right.
$$

Indeed, if equation (4) has a solution, then $w$ is defined on $[0, M(B(R), \rho)]$, and it follows that

$$
T_{f}(\lambda) \geq T_{f}(\lambda, p, R, \rho)>M(B(R), \rho)
$$


It is possible to give a more precise definition of $T_{f}(\lambda)$ :

$$
T_{f}(\lambda)=\max _{q \in] \lambda f(0), \lambda \bar{p}(f, \lambda, R, \rho)[} T_{f}(\lambda, q, R, \rho)
$$

where

$$
\bar{p}(f, \lambda, R, \rho)=\inf \left\{q>f(0) \mid T^{*}(q, f)<\lambda M(B(R), \rho)\right\}
$$

and $T^{*}(q, f)$ is the maximal existence time of the solution of

$$
\left\{\begin{array}{l}
\frac{d y}{d t}(t)=\max (f(y(t)), q) \\
y(0)=0
\end{array}\right.
$$

Indeed, let us consider a solution $w$ of equation (6) satisfying condition (7). According to Proposition 3, (ii),

$$
\frac{d w}{d \tau}(\tau) \geq \lambda f(w(\tau)) \quad \forall \tau \in[0, \psi(0)[
$$

and then

(a) $p=\frac{d w}{d \tau}(0) \geq \lambda f(0)$

(b) $\frac{d^{2} w}{d \tau^{2}} \geq 0$ implies that $\frac{d w}{d \tau}$ is increasing, and therefore

$$
\frac{d w}{d \tau}(\tau) \geq \frac{d w}{d \tau}(0)=p \quad \forall \tau \in[0, \psi(0)]
$$

Then $w(\tau)>g(\lambda \tau) \forall \tau \in[0, \psi(0)]$ and $\lambda M(B(R), \rho)<T^{*}\left(\frac{p}{\lambda}, f\right) . q \mapsto T^{*}(q, f)$ is decreasing, which ensures that

$$
\frac{p}{\lambda}<\bar{p}(f, \lambda, R, \rho)=\inf \left\{q>0 \mid T^{*}(q, f)<\lambda M(B(R), \rho)\right\}=\inf \left\{q>f(0) \mid T^{*}(q, f)<\lambda M(B(R), \rho)\right\}
$$

and it is not difficult to prove that

$$
T_{f}(\lambda)=\sup _{q \in] \lambda f(0), \lambda \bar{p}(f, \lambda, R, \rho)[} T_{f}(\lambda, q, R, \rho)
$$

is in fact a maximum. This provides a numerically usefull scheme (a shooting method) to solve equation (4) : for $q \in] \lambda f(0), \lambda \bar{p}(f, \lambda, R, \rho)]$, one solves equation (9), and then one tests condition (7) using the fact that

$$
q \mapsto \lambda \cdot \frac{\int_{0}^{\psi(0)}\left(\psi^{-1}(\tau)\right)^{N-1} \rho\left(\psi^{-1}(\tau)\right) f(w(\tau)) \cdot\left(\frac{d \psi}{d r}\left(\psi^{-1}(\tau)\right)\right)^{-1} d \tau}{\int_{0}^{\psi(0)}\left(\psi^{-1}(\tau)\right)^{N-1} \rho\left(\psi^{-1}(\tau)\right) \cdot\left(\frac{d \psi}{d r}\left(\psi^{-1}(\tau)\right)\right)^{-1} d \tau}
$$

is continuous.

(iii) Let us notice that the comparison of equation (1), when $\Omega$ is a bounded domain but not necessarily a ball, and $\rho$ is not necessarily radially symmetric, with the radial case only provides the well known result :

$$
\lambda_{c r}(\Omega, \rho) \geq \lambda_{c r}\left(\Omega^{*}, \rho^{*}\right)
$$

where $\Omega^{*}$ is the ball having the same volume as $\Omega$, and $\rho^{*}$ is obtained through Schwarz' symmetrisation method (see [B1-3], [HLP] or [Mos] for example). This is of course compatible with the fact that $M\left(\Omega^{*}, \rho^{*}\right) \geq$ $M(\Omega, \rho)$, but does not provide any further informations.

(iv) The results of this section (radial case) also apply to $\Omega=\mathbb{R}^{N}$ provided we are able to extend to $\mathbb{R}^{N}$ the symmetry results of B. Gidas, W-M. Ni and L. Nirenberg for a ball (see [GNN2], [Li], [LiN] ). 


\section{Comparison with other usual criteria}

We will compare the result of Theorem 1 with two other usual ways of estimating an upper bound for the critical explosion parameter.

The first one is commonly used : it relies on the comparison with the solution of the principal eigenvalue problem for the laplacian. It is more general (in the sense that if the assumptions of Theorem 1 apply, then we can also use it; the converse assertion would be false) provided the principal eigenvalue exists. But it does not take the nonlinearity well into account.

The second one relies on Pohozaev's method. It takes the nonlinearity very well into account, but there are many restrictions to the use of this method : $\rho$ is assumed to be constant on $\Omega$, and there are additional conditions on the domain $\Omega$. The use of Pohozaev's method is more technical than Theorem 1 , but the estimate of $\lambda_{\text {cr }}$ is sometimes optimal.

\subsection{A bound deduced from the comparison with a principal eigenvalue}

Let us consider equation (1) with the same assumptions as in Theorem 1. Let us define

$$
I(f)=\inf _{t>0} \frac{f(t)}{t}
$$

and $\lambda_{1}(\Omega)$ as the principal eigenvalue in $H_{0}^{1}(\Omega)$ of the eigenvalue problem

$$
-\Delta \phi=\lambda \cdot \rho(x) \phi(x) \quad(\lambda>0)
$$

Here $\Omega$ is not necessarily bounded, but $\Omega$ and $\rho$ are such that

$$
\begin{aligned}
\lambda_{1}(\Omega) & =\inf _{\substack{\varphi \in H_{0}^{1}(\Omega) \\
\varphi \neq 0}} \frac{\int_{\Omega}|\nabla \varphi|^{2}(x) d x}{\int_{\Omega} \rho(x)|\varphi|^{2}(x) d x} \\
& =\inf _{\varphi \in H_{0}^{1}(\Omega}\left\{\left.\int_{\Omega}|\nabla \varphi|^{2}(x) d x\left|\int_{\Omega} \rho(x)\right| \varphi\right|^{2}(x) d x=1\right\}
\end{aligned}
$$

exists. We assume for example that one of the following conditions is satisfied

(i) $\Omega$ is bounded and $\rho$ belongs to $L^{1}(\Omega)$ if $N=1$ or 2 , and to $L^{2 N /(N+2)}(\Omega)$ if $N \geq 3$

or

(ii) $\Omega=\mathbb{R}^{N}$ with $N \geq 3, \rho$ is smooth and such that there are two constants $K>0$ and $\alpha>1$ such that (see $[\mathrm{BCF}])$

$$
|\rho(x)| \leq K\left(1+|x|^{2}\right)^{-\alpha} \quad \forall x \in \mathbb{R}^{N}
$$

so that (for case (i) as well as for case (ii)) we know the existence of a strictly positive principal eigenvalue for the above problem.

Proposition 5 : $\quad$ Let $\Omega$ be a $C^{1}$ domain of $\mathbb{R}^{N}(N \geq 1), \rho$ a strictly positive $L_{\text {loc }}^{2}$ function on $\Omega$ such that $\lambda_{1}(\Omega)$ defined above exists, and assume that $f, \rho$ and $\Omega$ satisfy assumptions $(H 1)$ and (H2). Then, if $I(f)=\inf _{t>0} \frac{f(t)}{t}>0$, we have the following estimate

$$
\lambda_{c r} \leq \frac{\lambda_{1}(\Omega)}{I(f)}
$$

(For more details on that kind of results, see for example [B2,3], [JL]).

Proof of Proposition 5 : It is easy to prove that there exist $\phi_{1}$ in $H_{0}^{1}(\Omega)$, nonnegative, such that

$$
-\Delta \phi_{1}=\lambda_{1}(\Omega) \cdot \rho(x) \phi_{1}(x)
$$


using a variational argument : $\phi_{1}$ realizes the minimum

$$
\min _{\substack{\varphi \in H_{0}^{1}(\Omega) \\ \varphi \neq 0}} \frac{\int_{\Omega}|\nabla \varphi|^{2} d x}{\int_{\Omega} \rho|\varphi|^{2} d x}=\lambda_{1}(\Omega)
$$

Then, multiplying this equation by the solution $U$ of equation (1), and integrating by parts, we get

$$
\int_{\Omega}\left(\nabla U \cdot \nabla \phi_{1}\right) d x=\lambda_{1}(\Omega) \cdot \int_{\Omega} \rho U \phi_{1} d x
$$

and

$$
\int_{\Omega}\left(\nabla U \cdot \nabla \phi_{1}\right) d x=\lambda \cdot \int_{\Omega} \rho f(U) \phi_{1} d x \geq \lambda \cdot I(f) \int_{\Omega} \rho U \phi_{1} d x
$$

according to equation (1), the definition of $I$, and the fact that $U$ and $\phi_{1}$ are nonegative. This ensures that

$$
\lambda I(f) \leq \lambda_{1}(\Omega)
$$

\section{Remark 6 :}

(i) Let us notice that if

$$
T(f)<+\infty
$$

then

$$
\lim _{t \rightarrow+\infty} \frac{f(t)}{t}=+\infty
$$

and $I(f)$ is well defined : the assumptions of Proposition 5 can be deduced from the assumptions of Theorem 1. Theorem 1 is therefore less general than Proposition 5, provided $\lambda_{1}(\Omega)$ exists.

(ii) Theorem 1 takes the full nonlinearity into account, which is not the case for the criterion of Proposition 5. The bound given in the theorem is therefore better in some cases. For example, let us consider $f=f_{A}$ defined by

$$
\left\{\begin{array}{l}
f_{A}(t)=1 \quad(0 \leq t \leq A) \\
f_{A}(t)=e^{t-A} \quad(t \geq A)
\end{array}\right.
$$

Let us assume $N=1, \Omega=] 0,1[, \rho \equiv 1$. Then

$$
\frac{T\left(f_{A}\right)}{M(\Omega, \rho)}=8(A+1)<\left(\frac{\pi}{A}\right)^{2} \cdot A=\frac{\lambda_{1}(\Omega)}{I\left(f_{A}\right)} \quad \forall A>\frac{8}{\pi^{2}-8}
$$

\subsection{A bound deduced from Pohozaev's method}

Pohozaev's method (see [P]) is appliable to get an estimate of the critical explosion parameter when $\Omega$ is a bounded strictly star-shaped domain :

$$
\alpha(\Omega)=\sup _{\begin{array}{c}
x_{0} \in \Omega \text { such that } \\
\left(x-x_{0}\right) \cdot n(x)>0 \forall x \in \partial \Omega
\end{array}}\left(\inf \left\{\left(x-x_{0}\right) \cdot n(x) \mid x \in \partial \Omega\right\}\right)>0
$$

in the special case where $\rho$ is a constant function (we shall assume in the following that $\rho(x)=1 \quad \forall x \in \Omega$ ). Here, $n(x)$ is the outward pointing unit normal vector, for all $x \in \partial \Omega$. The main interest of this criterion relies on the fact that it clearly takes the full nonlinearity into account, and is therefore sometimes optimal (in dimension $N=2$ ). 
Proposition 7 : Let $\Omega$ be a bounded $C^{1}$ strictly star-shaped domain in $\mathbb{R}^{N}$ (with $N \geq 2$ ) such that $\alpha(\Omega)>0$. Let us consider equation (1) in the special case (autonomous case) of $\rho(x)=1 \forall x \in \Omega$

$$
-\Delta u_{\lambda}=\lambda \cdot f\left(u_{\lambda}\right)
$$

Then for any $\lambda \in] 0, \lambda_{\mathrm{cr}}\left[\right.$, for any solution $u_{\lambda}$, we have

$$
\lambda \leq \frac{2 N|\partial \Omega|}{\alpha(\Omega)} \cdot \frac{\int_{\Omega} F\left(u_{\lambda}(x)\right) d x}{\left(\int_{\Omega} f\left(u_{\lambda}(x)\right) d x\right)^{2}}
$$

where $F(t)=\int_{0}^{t} f(s) d s$.

One can also make use of Pohozaev's method in the case of unbouded domains, but it is a little bit more technical because one has to be careful with the integrations by parts (for instance, see [EL1,2]).

Proof of Proposition 7 : The proof is easily obtained through Rellich's identity (see $[R],[P]$ )

$$
\int_{\partial \Omega} \frac{1}{2}\left(x-x_{0}\right) \cdot n(x)\left|\nabla u_{\lambda}\right|^{2} d \sigma(x)+\frac{N-2}{2} \int_{\Omega}|\nabla u|^{2} d x=\lambda N \int_{\Omega} F\left(u_{\lambda}(x)\right) d x
$$

To get Rellich's identity, one has to multiply equation (1) by $\left(x-x_{0}\right) \cdot \nabla u_{\lambda}$, and integrate by parts. Then

$$
\int_{\partial \Omega} \frac{1}{2}\left(x-x_{0}\right) \cdot n(x)\left|\nabla u_{\lambda}\right|^{2} d \sigma(x) \leq \lambda N \int_{\Omega} F\left(u_{\lambda}(x)\right) d x
$$

because $N \geq 2$. We assume now - to simplify a little bit - that there exists an $x_{0} \in \Omega$ realizing $\alpha(\Omega)$ i.e. such that

$$
\alpha(\Omega)=\inf \left\{\left(x-x_{0}\right) \cdot n(x) \mid x \in \partial \Omega\right\}
$$

Using successively the definition of $\alpha(\Omega)$, the fact that

$$
\nabla u_{\lambda}(x)=\left(\nabla u_{\lambda}(x) \cdot n(x)\right) n(x) \quad \forall x \in \partial \Omega
$$

because $u_{\lambda}$ is constant on $\partial \Omega$, Hölder's inequality, an integration by parts, and the definition of $u_{\lambda}$, we get

$$
\begin{aligned}
\int_{\partial \Omega} \frac{1}{2}\left(x-x_{0}\right) \cdot n(x)\left|\nabla u_{\lambda}\right|^{2} d \sigma(x) \geq & \frac{\alpha(\Omega)}{2} \int_{\partial \Omega}\left|\nabla u_{\lambda}\right|^{2} d \sigma(x) \\
=\frac{\alpha(\Omega)}{2} \int_{\partial \Omega}\left|\nabla u_{\lambda} \cdot n(x)\right|^{2} d \sigma(x) & \\
\geq & \frac{\alpha(\Omega)}{2|\partial \Omega|}\left(\int_{\partial \Omega} n(x) \cdot \nabla u_{\lambda} d \sigma(x)\right)^{2} \\
& =\frac{\alpha(\Omega)}{2|\partial \Omega|}\left(\int_{\Omega}-\Delta u_{\lambda} d x\right)^{2} \\
& =\frac{\alpha(\Omega)}{2|\partial \Omega|} \lambda^{2}\left(\int_{\Omega} f\left(u_{\lambda}(x)\right) d x\right)^{2}
\end{aligned}
$$

which proves the result.

Remark 8 : To apply Pohozaev's method, one has to impose stronger assumptions than in Theorem $1(\Omega$ is a bounded strictly star-shaped domain, $\rho \equiv 1$ ), but one uses the full nonlinearity, and in dimension $N=2$, 
this method is sometimes optimal (which is never the case for Theorem 1). Let us consider for instance (see [CLMP], [G], [JL], [Lio]) $f(t)=e^{t}$ when the domain is $\Omega=\left\{x \in \mathbb{R}^{2}:|x|^{2}<1\right\}$. Then

$$
\psi_{\mu}(r)=-2 \ln \left(\frac{\mu+r^{2}}{\mu+1}\right) \quad(\mu>0)
$$

solution of equation (1) with

$$
\lambda=\lambda(\mu)=\frac{8 \mu}{(\mu+1)^{2}}
$$

The condition deduced from Pohozaev's method is now

$$
\lambda \leq \frac{4 \mu}{\mu+1}
$$

The equality is realized if and only if $\lambda=2$ (i.e. $\mu=1$ ) which corresponds to

$$
\lambda=\lambda_{c r}=2
$$

\section{Conclusion}

As a conclusion, we will just mention some possible extensions.

(i) The method could be generalized to more general elliptic operators than the Laplacian provided that they satisfy a Maximum Principle (see [BNV] for a general review of the different forms of generalized Maximum Principles).

(ii) The method also apply to nonhomogeneous Dirichlet conditions, i.e. to the problem

$$
\left\{\begin{array}{l}
-\Delta U=\lambda \cdot \rho(x) f(U) \quad(x \in \Omega) \\
U_{\mid \partial \Omega}=\Phi
\end{array}\right.
$$

provided $\Phi$ is a $L^{\infty}(\partial \Omega)$ bounded function.

(iii) The inequality

$$
y(\lambda M(\Omega, \rho)) \leq\|u\|_{L^{\infty}(\Omega)}
$$

given in Lemma 2 is an equality if $f$ is constant on a neighbourhood of $0+$. When $\Omega$ is a ball, $f$ is constant on a neighbourhood of $0+$ if and only if

$$
y(\lambda M(\Omega, \rho))=\|u\|_{L^{\infty}(\Omega)}
$$

for $\lambda$ large enough (see Remark 4 (iii)). This result has to be generalized to the case of general domains $\Omega$.

(iv) In the demonstration of Theorem 1, we were using the usual Maximum Principle on a $C^{1}$ bounded domain (that is why we assumed a so strong condition $(H 2)$ on the regularity of the solution $\psi$ of equation (2). The recent work of H. Beresticky, L. Nirenberg and S.R.S. Varadhan (see [BNV]) on the Maximum Principle for second order elliptic operators in general domains allows us to relax a little bit this assumption and work with less regular solutions of equation (2).

Acknowledgements The authors thank J. Mossino for usefull comments on their work. 


\section{References}

[ADN] Agmon S, Douglis S, Nirenberg L. Estimates near the boundary for solutions of elliptic partial differential equations satisfying general boundary value conditions I. Comm. Pure Appl. Math. 12 (1959) 623-727 II. Comm. Pure Appl. Math. 17 (1964) 35-92

[A1] Amann H. On the existence of positive solutions of nonlinear elliptic boundary value problems. Indiana Univ. Math. J. 21 (1971/72) 125-146

[A2] Amann H. Fixed point equations and nonlinear eigenvalue problems in ordered Banach spaces. SIAM Rev. 18 no. 4 (1976) 620-709

[B1] Bandle C. Isoperimetric inequalities and applications. Pitman, Boston, London, Melbourne (1980)

[B2] Bandle C. Bounds for the solutions of Poisson problems and application to nonlinear eigenvalue problems. SIAM J. Math. Appl. Anal.6 (1975) 146-152

[B3] Bandle C. Existence theorems, qualitative results and apriori bounds for a class of nonlinear Dirichlet problems. Arch. Rat. Mech. Anal. 58 (1975) 219-238

[BNV] Beresticky H, Nirenberg L, Varadhan SRS. The principal eigenvalue and the Maximum Principle for second order elliptic operators in general domains. preprint LMENS 92-14. To appear in Communications on Pure and Applied Mathematics.

[BCF] Brown KJ, Cosner C, Fleckinger J. Principal eigenvalue for problems with indefinite weight functions on $\mathbb{R}^{N}$. Proc. Amer. Math. Soc. 109 no.1 (1990) 147-155

[CLMP] Caglioti E, Lions PL, Marchioro C, Pulvirenti M. A special class of stationary flows for twodimensional Euler equations : a statistical mechanics description. Comm. Math. Phys. 143 (1992) 501-525

[CR] Crandall MG, Rabinowitz PH. Some continuation and variational methods for positive solutions of nonlinear elliptic eigenvalue problems. Arch. Rat. Mech. Anal. 58 (1975) 207-218

[EL1] Esteban MJ, Lions PL. Non-existence de solutions non-nulles pour des problèmes semilinéaires dans des ouverts non-bornés. C.R. Acad. Sci. Paris 290 (1980) 1083-1085

[EL2] Esteban MJ, Lions PL. Existence and nonexistence results for semilinear elliptic problems in unbounded domains. Proc. Roy. Soc. Ed. 93A (1982/83) 1-14

[FLN1] de Figueiredo D, Lions PL, Nussbaum RD. Estimations a priori pour les solutions positives de problemes elliptiques semilineaires. C.R. Acad. Sci. Paris 290 (1980) 211-220

[FLN2] de Figueiredo D, Lions PL, Nussbaum RD. A priori estimates and existence results for positive solutions of semilinear elliptic equations. J. Math. Pures Appl. (9) 61 no. 1 (1982) 41-63

[G] Gelfand IM. Some problems in the theory of quasi-linear equations. Amer. Math. Soc. Transl. 29 (1963) 295-381

[GNN1] Gidas B, Ni W-M, Nirenberg L. Symmetry and related properties via the maximum principle. Comm. Math. Phys. 68 (1979) 209-243

[GNN2] Gidas B, Ni W-M, Nirenberg L. Symmetry of positive solutions of nonlinear elliptic equatins in $\mathbb{R}^{N}$. Math. Anal. Appl. (coll. Adv. in Math. Suppl. Stud. 7a), Part A, L. Nachbin (ed.), Academic Press (1981) 370-401

[GS] Gidas B, Spruck J. Global and local behaviour of positive solutions of nonlinear elliptic equations. Comm. Pure Appl. Math. 34 (1981) 525-598

[HLP] Hardy GH, Littlewood JE, Polya G. Inequalities. Cambridge university Press, Cambridge (1967)

[IL] Ishii H, Lions PL. Viscosity solutions of fully nonlinear second-order elliptic partial differential equations. J. Diff. Eq. 83 (1990) 26-78

[J] Joseph DD. Bounds on $\lambda$ for positive solutions of $\Delta \psi+\lambda f(r)\{\psi+G(\psi)\}=0$.. Q. Appl. Math. 23 (1966) 349-354

[JL] Joseph DD, Lundgren TS. Quasilinear problems driven by positive sources. Arch. Rat. Mech. Anal. 49 (1973) 241-269

[KC] Keller HB, Cohen DS. Some positive problems suggested by nonlinear heat generation. J. Math. Mech. 16 (1967) 1361-1376

[Li] Li C. Monotonicity and symmetry of solutions of fully nonlinear elliptic equations on unbounded domains. Comm. P.D.E. 16 (1991) 585-615

[LiN] Li Y, Ni W-M. Radial symmetry of positive solutions of nonlinear elliptic equations in $\mathbb{R}^{N}$. Comm. P.D.E. 18, no. $5 \& 6$ (1993) 1043-4054 
[Lio] Lions PL. On the existence of positive solutions of semilinear elliptic equations. SIAM Rev. 24 no.4 (1982) 441-467

[Mor] Morrey C. Multiple integrals in the calculus of variations. Springer Verlag, New-York (1966)

[Mos] Mossino J. Inégalités isopérimétriques et applications en physique. Travaux en cours, Hermann, Paris (1984)

[P] Pohozaev. Eigenfunctions of the equation $\Delta u+\lambda f(u)=0$. Soviet. Math. Dokl. 5 (1965) 1408-1411

[R] Rellich F. Darstellung der Eigenwerte von $\Delta u+\lambda u=0$ durch ein Rand integral. Math. Z. 46 (1940) $635-636$ 